\title{
Numerical Time-Domain Modeling of Lamb Wave Propagation Using Elastodynamic Finite Integration Technique
}

\author{
Hussein Rappel, ${ }^{1}$ Aghil Yousefi-Koma, ${ }^{1}$ Jalil Jamali, $^{2}$ and Ako Bahari ${ }^{3}$ \\ ${ }^{1}$ Center of Advanced Systems and Technologies (CAST), School of Mechanical Engineering, College of Engineering, \\ University of Tehran, Tehran 14399 57131, Iran \\ ${ }^{2}$ Department of Mechanical Engineering, Islamic Azad University, Shushtar Branch, Shushtar, Iran \\ ${ }^{3}$ School of Railway Engineering, Iran University of Science and Technology, Tehran 16846 13114, Iran \\ Correspondence should be addressed to Aghil Yousefi-Koma; aykoma@ut.ac.ir
}

Received 19 October 2012; Accepted 19 November 2012; Published 10 July 2014

Academic Editor: Hamid Mehdigholi

Copyright (C) 2014 Hussein Rappel et al. This is an open access article distributed under the Creative Commons Attribution License, which permits unrestricted use, distribution, and reproduction in any medium, provided the original work is properly cited.

\begin{abstract}
This paper presents a numerical model of lamb wave propagation in a homogenous steel plate using elastodynamic finite integration technique (EFIT) as well as its validation with analytical results. Lamb wave method is a long range inspection technique which is considered to have unique future in the field of structural health monitoring. One of the main problems facing the lamb wave method is how to choose the most appropriate frequency to generate the waves for adequate transmission capable of properly propagating in the material, interfering with defects/damages, and being received in good conditions. Modern simulation tools based on numerical methods such as finite integration technique (FIT), finite element method (FEM), and boundary element method (BEM) may be used for modeling. In this paper, two sets of simulation are performed. In the first set, group velocities of lamb wave in a steel plate are obtained numerically. Results are then compared with analytical results to validate the simulation. In the second set, EFIT is employed to study fundamental symmetric mode interaction with a surface braking defect.
\end{abstract}

\section{Introduction}

Lamb wave testing technique is increasingly used for assessing defects in thin-wall structures like plate and pipes [13]. Lamb waves are elastic waves whose wavelength is in the same order as thickness of the structure [4]. One of the main advantages of lamb wave technique is that it allows long-range inspection in contrast to traditional ultrasonic testing, where the coverage is limited to a small area in vicinity of each transducer. Lamb waves were first described theoretically by Horace Lamb in 1917 [5]. These waves arise from coupling between shear and longitudinal waves reflected at the top and bottom edges of a thin wall structure [6]. Lamb wave theory can be found in a number of text books [7]. Defects such as corrosion and fatigue cracks cause changes in effective thickness and local material properties and therefore measurement of variations in lamb wave propagation can be used to assess the integrity of plate [1]. Successful usage of lamb waves in an inspection system needs to understand its schemes of propagation in a waveguide and its scattering at defects. Thus, there is an increasing demand for powerful, flexible, and accurate simulation techniques. First works on numerical simulation of ultrasonic waves were done by Harumi (1986) and Yamawaki and Saito (1992) who calculated and visualized bulk wave propagation [8]. Now, numerical simulation of lamb waves is possible. Common techniques which are used to simulate lamb wave propagation are finite difference time domain (FDTD) [9], finite element method (FEM) [5], boundary element method (BEM) [10], elastodynamic finite integration technique (EFIT) [11, 12], and specialized methods for guided wave calculations such as hybrid methods [13] and semianalytical finite element method (SAFEM) [8].

In this work, calculations are based on elastodynamic finite integration technique; historically, finite integration technique was introduced by Weiland in electrodynamics. Fellinger and Langenberg used Weiland's idea for governing equations of ultrasonic waves in solid, calling it EFIT [14]. EFIT is a grid based numerical time-domain method, using velocity-stress formalism, and easily treats with different 
boundary conditions which are essential to model ultrasonic wave propagation [12]. Because of its relative simplicity and flexibility, Schubert et al. used EFIT equations to cylindrical coordinates (CEFIT) to simulate axisymmetric wave propagation in pipes with a $2 \mathrm{D}$ grid [15]. Schubert also used finite integration technique to simulate elastic wave propagation in porous concrete and showed efficiency of EFIT to model a diverse range of applications [16].

Two sets of simulation results are presented in this work using a program developed in MATLAB environment. In the first one, lamb wave propagation in a $2 \mathrm{D}$ steel plate is discussed. Results are then compared with analytical results to validate the accuracy of modeling and, in the second example, interaction lamb wave with a surface breaking defect is investigated.

\section{The Elastodynamic Finite Integration Technique for Linear Elastics}

2.1. Governing Equations. The governing equations of elastic waves in a general media are the Cauchy equation of motion and equation of deformation rate. These equations are given in integral form for a finite volume $V$ and surface $S$ as follows:

$$
\begin{gathered}
\frac{\partial}{\partial t} \int_{V} \rho v_{i} d V=\int_{S} T_{i j} n_{i} d S+\int_{V} f_{i} d V, \\
\frac{\partial}{\partial t} \int_{V} s_{i j k l} T_{k l} d V=\frac{1}{2} \int_{S}\left(v_{i} n_{j}+v_{j} n_{i}\right) d S,
\end{gathered}
$$

where $v$ is the particle velocity vector, $T$ is stress tensor, $\rho$ is density, $n$ is the outward normal vector on surface $S, f$ is the body force vector, and $s$ is the compliance tensor. The inverse of $s$ is the stiffness tensor $c$. Thus, using stiffness tensor, deformation rate equation can be expressed in another form. Consider

$$
\frac{\partial}{\partial t} \int_{V} T_{k l} d V=\frac{1}{2} \int_{S} c_{k l i j}\left(v_{i} n_{j}+v_{j} n_{i}\right) d S=\int_{S} c_{k l i j} v_{i} n_{j} d S .
$$

In the case of isotropic material $c$ can be written as [17]

$$
c_{i j k l}=\lambda \delta_{i j} \delta_{k l}+\mu\left(\delta_{i k} \delta_{j l}+\delta_{i l} \delta_{j k}\right)
$$

where $\lambda$ and $\mu$ are lame constants.

2.2. Spatial Discretized Form of Two Dimensional EFIT. Consider the Cartesian coordinate $\{x, y, z\}$ and ultrasonic wave which propagates in two dimensional $x z$-plane. To apply FIT to (1) and (2), squares shown in Figure 1 are used as integral volume $V$, assuming constant $v$ and $T$ for each volume.

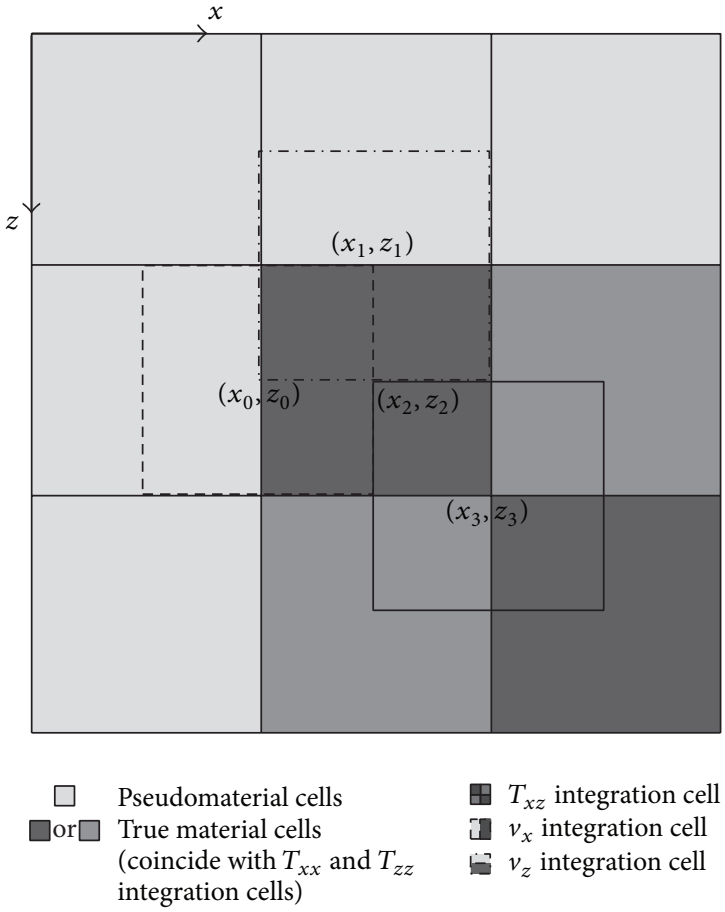

FIGURE 1: Definition of integration cells for stress and velocity components. The geometry consists of four true material cells and four pseudomaterial cells [12].

The final results for discretized form are

$$
\begin{aligned}
& \frac{\partial v_{x}\left(x_{0}, z_{0}\right)}{\partial t} \\
& =\frac{1}{\rho}\left[\frac{T_{x x}\left(x_{0}+(\Delta x / 2), z_{0}\right)-T_{x x}\left(x_{0}-(\Delta x / 2), z_{0}\right)}{\Delta x}\right. \\
& \quad+\frac{T_{x z}\left(x_{0}, z_{0}+(\Delta z / 2)\right)-T_{x z}\left(x_{0}, z_{0}-(\Delta z / 2)\right)}{\Delta z} \\
& \left.\quad+\frac{f_{x}}{\Delta x \Delta z}\right] .
\end{aligned}
$$

A same manner of integration equation (1) about a $v_{z}$ integration cell centered at $\left(x_{1}, z_{1}\right)$ results in

$$
\begin{aligned}
& \frac{\partial v_{z}\left(x_{1}, z_{1}\right)}{\partial t} \\
& =\frac{1}{\rho}\left[\frac{T_{x z}\left(x_{1}+(\Delta x / 2), z_{1}\right)-T_{x z}\left(x_{1}-(\Delta x / 2), z_{1}\right)}{\Delta x}\right. \\
& \quad+\frac{T_{z z}\left(x_{1}, z_{1}+(\Delta z / 2)\right)-T_{z z}\left(x_{1}, z_{1}-(\Delta z / 2)\right)}{\Delta z} \\
& \left.\quad+\frac{f_{z}}{\Delta x \Delta z}\right] .
\end{aligned}
$$




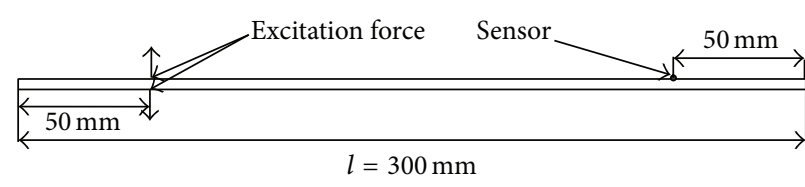

(a)

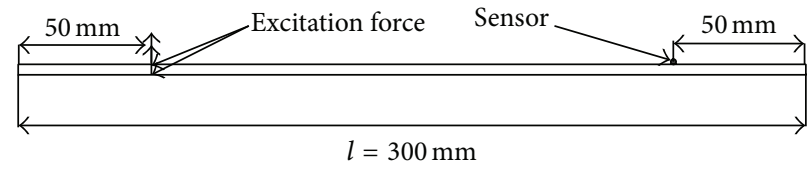

(b)

FIGURE 2: Steel sheet with length $l=300 \mathrm{~mm}$ and thickness $d=2 \mathrm{~mm}$. (a) Excitation pattern for symmetric mode. (b) Excitation pattern for axisymmetric mode.

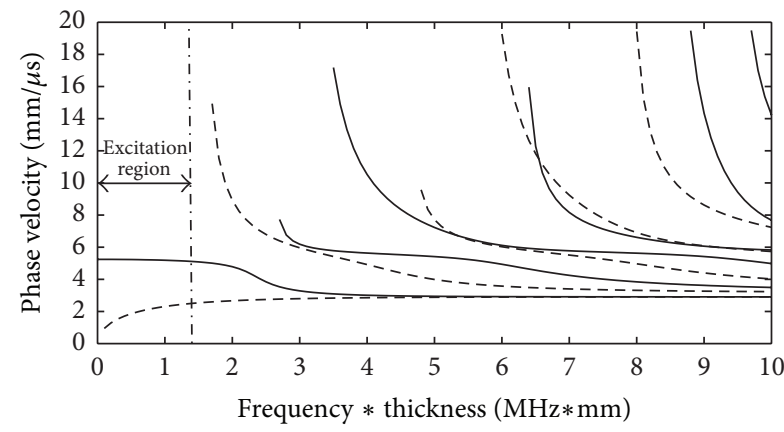

(a)

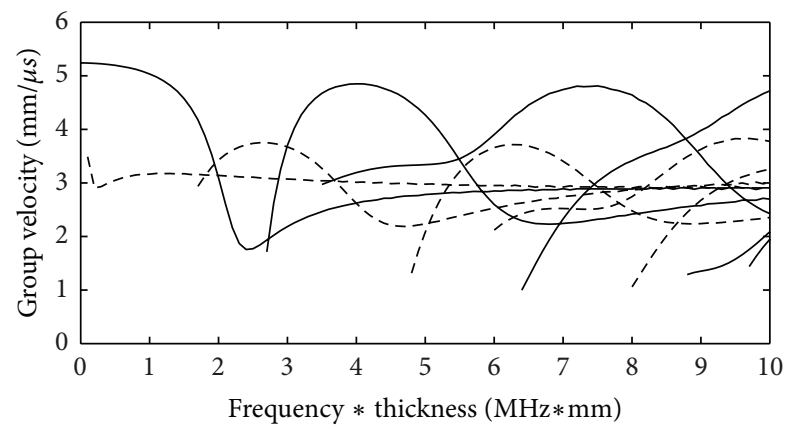

(b)

FIgURE 3: Dispersion diagram for a plate steel (a) phase velocity curve and (b) group velocity curve.

Now, using the normal stress equations, integration of (3) about $T_{x x}$ and $T_{z z}$ centered at $\left(x_{2}, z_{2}\right)$ yields

$$
\begin{aligned}
& \frac{\partial T_{x x}\left(x_{2}, z_{2}\right)}{\partial t} \\
& =(\lambda+2 \mu)\left[\frac{v_{x}\left(x_{2}+(\Delta x / 2), z_{2}\right)-v_{x}\left(x_{2}-(\Delta x / 2) \cdot z_{2}\right)}{\Delta x}\right] \\
& \quad+\lambda\left[\frac{v_{z}\left(x_{2}, z_{2}+(\Delta z / 2)\right)-v_{z}\left(x_{2}, z_{2}-(\Delta z / 2)\right)}{\Delta z}\right], \\
& \frac{\partial T_{z z}\left(x_{2}, z_{2}\right)}{\partial t} \\
& =(\lambda+2 \mu)\left[\frac{v_{z}\left(x_{2}, z_{2}+(\Delta z / 2)\right)-v_{z}\left(x_{2}, z_{2}-(\Delta z / 2)\right)}{\Delta z}\right] \\
& \quad+\lambda\left[\frac{v_{x}\left(x_{2}+(\Delta x / 2), z_{2}\right)-v_{z}\left(x_{2}-(\Delta x / 2), z_{2}\right)}{\Delta x}\right] .
\end{aligned}
$$

Finally, integration of (3) over $T_{x z}$ integration cell centered at $\left(x_{3}, z_{3}\right)$ the intersection for material cells results in

$$
\begin{aligned}
& \frac{\partial T_{x z}\left(x_{3}, z_{3}\right)}{\partial t} \\
& =\mu\left[\frac{v_{x}\left(x_{3}, z_{3}+(\Delta z / 2)\right)-v_{x}\left(x_{3}, z_{3}-(\Delta z / 2)\right)}{\Delta z}\right. \\
& \left.\quad+\frac{v_{z}\left(x_{3}+(\Delta x / 2), z_{3}\right)-v_{z}\left(x_{3}-(\Delta x / 2), z_{3}\right)}{\Delta x}\right] .
\end{aligned}
$$

As shown in Figure 1, to simplify indexing into stress and velocity arrays of staggered grids when programming the numerics and to keep the same array sizes for all quantities, pseudomaterial cells are used. These cells have the same material properties as the true material they are added to but are not part of physical simulations.

2.3. Time Discretization. Central differences are used to discretize the equations in time domain which results in the velocity and stress components being staggered in time by $\Delta t / 2[15]$. Consider

$$
\begin{gathered}
v^{n}=v^{n-1}+\Delta t i^{n-(1 / 2)}, \\
T^{n+(1 / 2)}=T^{n-(1 / 2)}+\Delta t \dot{T}^{n},
\end{gathered}
$$

where $\Delta t$ is time interval, superscript $n$ is integer number of time step, and dot $\{\cdot\}$ denotes the time differentiation.

Equations (5)-(8) are solved at all points in simulation space and, by use of (9), the simulation proceeds in time in a "leap frogging" manner. A specific stability condition and adequate spatial resolution must be satisfied to guarantee EFIT convergence and accurate answers [15].

\section{Propagation of Lamb Wave in a Steel Plate}

In this part, the propagation of lamb wave in a steel plate is simulated using 2D-EFIT. The steel plate has the length $l=$ $300 \mathrm{~mm}$ and the thickness $d=2 \mathrm{~mm}$. Table 1 shows material properties used in this paper.

As excitation source, point sources at top and bottom borders of plate are used. Figure 2 shows location of applied loads.

Using excitation patterns shown in Figure 2 and dispersion diagram for steel plate (Figure 3), single mode lamb wave is generated which makes signal interpretation easier. 


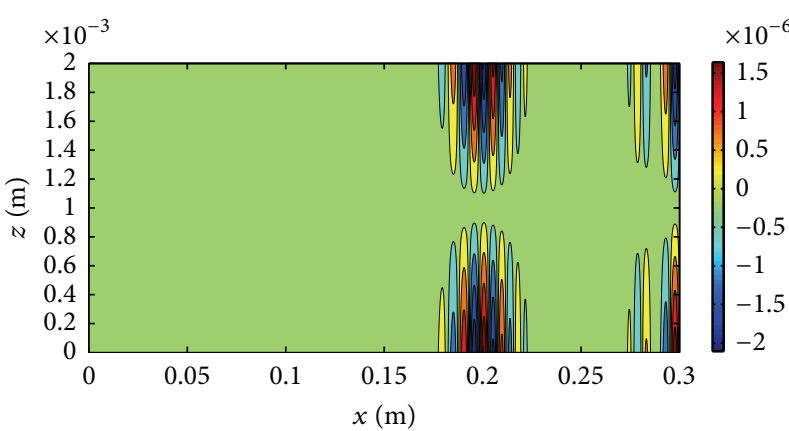

(a)

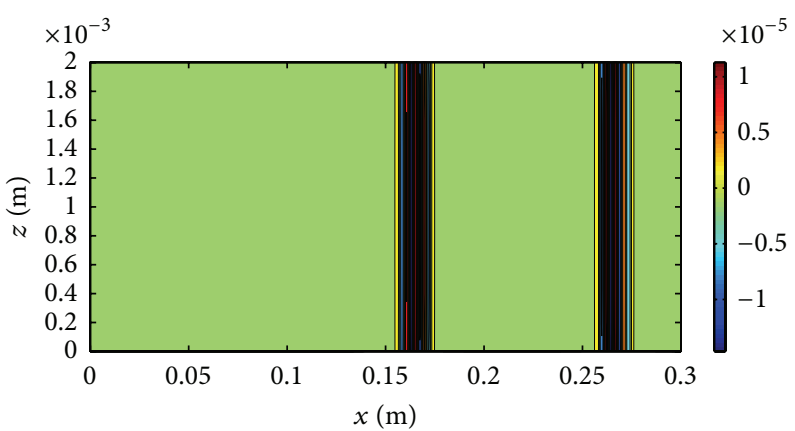

(b)

FIGURE 4: Lamb wave propagation in a steel plate at time $T=80 \mu \mathrm{s}$. The snapshot represents normal component of particle velocity $\left(v_{z}\right)$ : $(\mathrm{a})$ symmetric mode and (b) axisymmetric mode.

TABLE 1: Material properties used for simulation.

\begin{tabular}{lc}
\hline Property & Value \\
\hline Density & $7700\left(\mathrm{~kg} / \mathrm{m}^{3}\right)$ \\
Elastic modulus & $195 \mathrm{Gpa}$ \\
Lame constant $\lambda$ & $96.95 \mathrm{Gpa}$ \\
Lame constant $\mu$ & $76.17 \mathrm{Gpa}$ \\
\hline
\end{tabular}

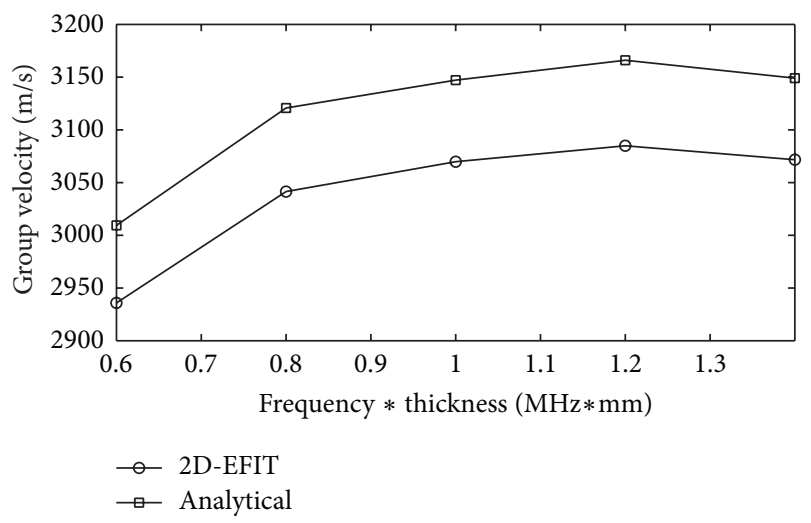

FIGURE 5: Analytical group velocities comparison with 2D-EFIT results for fundamental axisymmetric mode.

Using 2D-EFIT code developed in MATLAB, propagation of lamb wave in the steel plate is simulated. To guarantee stability and accuracy of results, $\Delta x$ and $\Delta z$ are chosen $0.2 \mathrm{~mm}$ and $\Delta t$ is $20 \mathrm{~ns}$. The simulation results using EFITtool for symmetric and axisymmetric modes are presented in Figure 4, where the ultrasonic wave field in the plate at time $T=80 \mu$ s is shown (excitation pulse is a raised cosine with five cycles with center frequency of $500 \mathrm{kHz}$ ).

As shown in Figure 4, for the fundamental symmetric mode $\left(S_{0}\right)$, the lamb wave field is symmetric about half plane line and, for the fundamental axisymmetric mode $\left(A_{0}\right)$, normal component of particle velocity $v_{z}$ has the same value for every particle with same longitudinal position. From dispersion curve, we find that $S_{0}$ travels faster than $A_{0}$ which is validated by simulation results (see Figure 4 ).

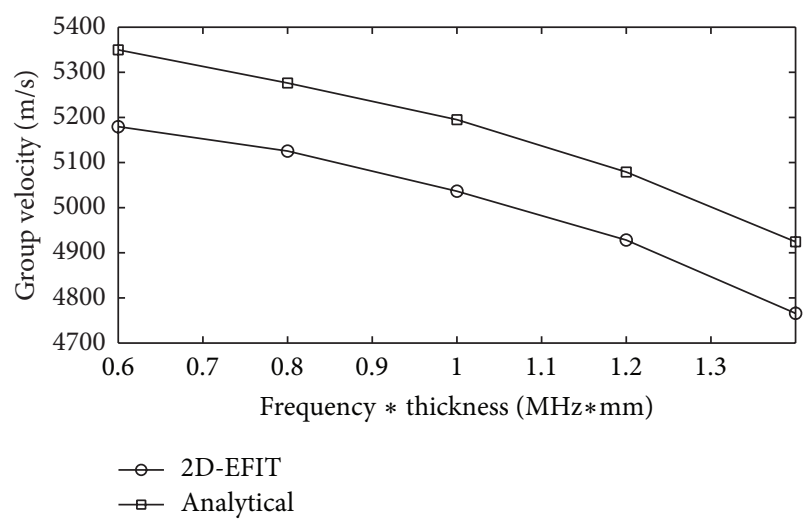

FIGURE 6: Analytical group velocities comparison with 2D-EFIT results for fundamental symmetric mode.

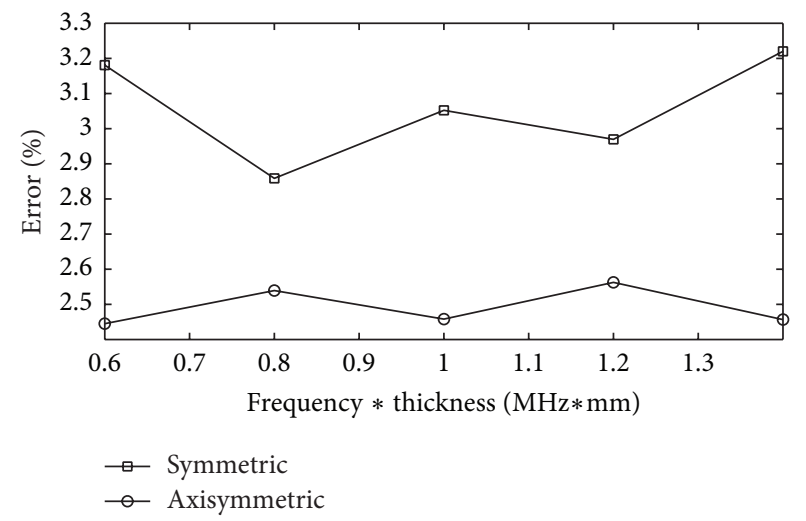

FIGURE 7: Error comparison for symmetric and axisymmetric modes.

In order to check EFIT accuracy, group velocities obtained from simulation are compared with analytical results at both symmetric and axisymmetric modes (Figures 5 and 6 ).

Figures 5-7 show good agreement for simulation results with analytical ones; also Figure 7 shows error dependence 


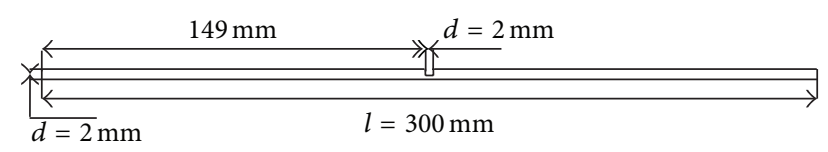

FIGURE 8: Schematic of model used for studying interaction lamb wave with a defect in a steel plate.

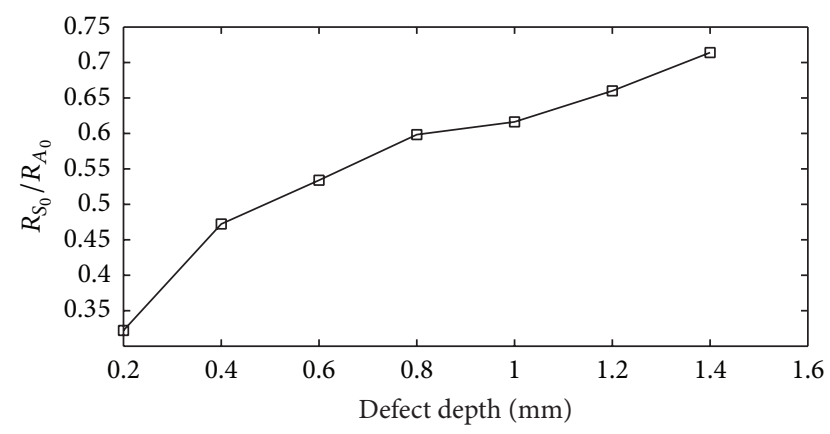

FIGURE 9: Ratio of reflection coefficients as function of crack depth.

on frequency for axisymmetric mode is less than symmetric mode.

\section{Reflection of the Fundamental Symmetric Mode $\left(S_{0}\right)$ from a Defect}

In this section, interaction of the $S_{0}$ mode with a defected steel plate is analyzed. The results presented here were used for a sizing study of rectangular surface braking defect with different depths and opening length $2 \mathrm{~mm}$ on a steel plate (Figure 8).

The same method used in the proceeding section is used to generate single mode with center frequency of $500 \mathrm{kHz}$. However, as the lamb wave interacts with a defect, the axisymmetric mode will be generated. To study lamb wave interaction with a defect, the ratio of the maximum amplitudes for two modes $R_{S_{0}} / R_{A_{0}}$ is then calculated and compared at different depths (Figure 9).

Figure 10 shows the ultrasonic wave field in the defected plate at time $T=60 \mu \mathrm{s}$; the defect depth is $0.4 \mathrm{~mm}$. As shown in Figure 10, because symmetric modes travel faster than axisymmetric ones, mode separation happens after lamb wave interaction with defect.

\section{Conclusion}

EFIT was used for studying lamb wave propagation in a steel plate using a program developed in MATLAB environment. Two sets of simulation results were presented in this paper. In the first example, group velocities of lamb wave for different frequencies were obtained using numerical signals and then the results were compared with analytical results; the comparison shows, for both fundamental symmetric and axisymmetric modes, the group velocity values are in good agreement with theoretical ones. In the second example, reflection of $S_{0}$ mode from a defect is studied and ratio of

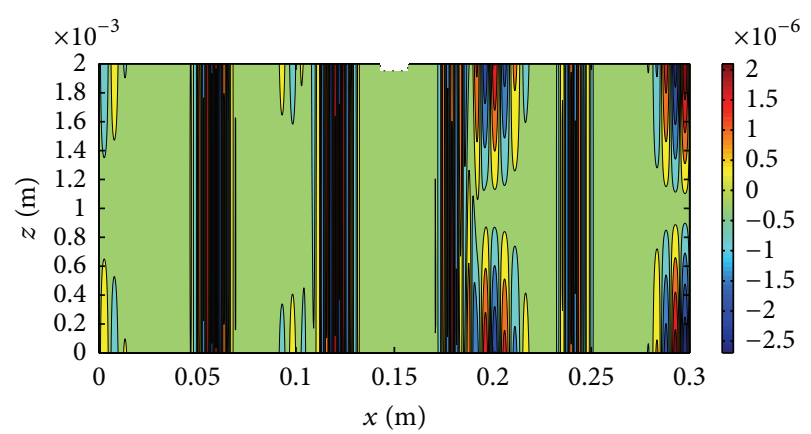

FIgURE 10: Lamb wave propagation in a steel plate with defect at time $T=60 \mu$ s. The snapshot represents normal component of particle velocity $\left(v_{z}(\mathrm{~m} / \mathrm{s})\right)$.

reflection coefficients was obtained as a function of crack depth which shows that as the crack depth increases the ratio $R_{S_{0}} / R_{A_{0}}$ increases. Each calculation presented in this paper was done on ordinary PC (Core i5, 2.4 GHz, 4 GB RAM).

\section{Conflict of Interests}

The authors declare that there is no conflict of interests regarding the publication of this paper.

\section{References}

[1] J. G. Yu, F. E. Ratolojanhary, and J. E. Lebvre, "Guided waves in functionally graded viscoelastic plates," Journal of Composite Structures, vol. 93, pp. 2671-2677, 2011.

[2] V. T. Rathod and D. R. Mahapatra, "Ultrasonic lamb wave based monitoring of corrosion type of damage in plate using a circular array of piezoelectric transducer," NDT \& E International, vol. 44, no. 7, pp. 628-636, 2011.

[3] A. Raghavan and C. E. S. Cesnik, "Review of guided wave structural health monitoring," Journal of The Shock and Vibration Digest, vol. 39, pp. 91-113, 2007.

[4] C. M. Yeum, H. Sohn, and J. B. Ihn, "Lamb wave mode decomposition using concentric ring and circular piezoelectric transducers," Wave Motion, vol. 48, no. 4, pp. 358-370, 2011.

[5] S. Sorohan, N. Constantin, M. Gavan, and V. Anghel, "Extraction of dispersion curves for waves propa-gating in free complex waveguides by standard finite element codes," Ultrasonics, vol. 51, pp. 503-515, 2011.

[6] V. B. Yadav, T. Piralima, V. Raghuram, and N. N. Kishore, "A finite difference simulation of multi-mode lamb waves in aluminium sheet with experimental verification using laser based ultrasonic generation," in Proceedings of the 12th AsiaPacific conference on NDT, Aukland, New Zeland, November 2006.

[7] K. F. Graff, Wave Motion on Elastic Solids, Dover Publications, New York, NY, USA, 1991.

[8] T. Hayashi and J. L. Rose, "Guided wave simulation and visualization by a semianalytical finite element method," Journal of Materials Evaluation, vol. 61, pp. 75-79, 2003.

[9] D. Gsell, T. Leutenegger, and J. Dual, "Modeling threedimensional elastic wave propagation in circular cylindrical structures using a finite-difference approach," Journal of Acoustic Society of America, vol. 116, no. 6, pp. 3284-3293, 2004. 
[10] Z. Xiaoliang and J. L. Rose, "Boundary element modeling for defect characterization potential in a wave guide," International Journal of Solid and Structures, vol. 40, pp. 2645-2658, 2003.

[11] C. A. C. Leckey, M. D. Rogge, C. A. Miller, and M. K. Hinders, "Multiple-mode lamb wave scattering simu-lations using 3D elastodynamic finite integration technique," Journal of Ultrasonics, vol. 52, pp. 193-207, 2012.

[12] D. C. Calvo, K. E. Rudd, M. Zampolli, W. M. Sanders, and L. D. Bibee, "Simulation of acoustic scattering from an aluminum cylinder near a roughinterface using the elastodynamic finite integration technique," Wave Motion Journal, vol. 47, pp. 616634, 2010.

[13] Y. Cho, D. Hongerholt, and J. L. Rose, "Lamb wave scattering analysis for reflector characterization," IEEE Transactions on Ultrasonics, vol. 44, pp. 44-52, 1997.

[14] R. Marklein, "The finite integration technique as a general tool to computeacoustic, electromagnetic, elastodynamic, and coupled wave fields," in Review of Radio Science: 1999-2002 URSI, W. Stone, Ed., IEEE Press and John Wiley and Sons, New York, NY, USA, 2002.

[15] F. Schubert, A. Peiffer, B. Kohler, and T. Sanderson, “The elastodynamic finite integration technique for waves in cylindrical geometries," The Journal of the Acoustical Society of America, vol. 104, no. 5, pp. 2604-2614, 1998.

[16] F. Schubert and B. Koehler, “Three-dimensional time domain modeling of ultrasonic wave propagation in concrete in explicit consideration of aggregates and porosity," Journal of Computational Acoustics, vol. 9, no. 4, pp. 1543-1560, 2001.

[17] K. Takata, K. Nakahata, F. Schubert, and B. Kohler, "Imagebased FIT modeling for coupled elastody-namic and acoustic problems," in Proceedings of AIP Conference, vol. 1335, pp. 720727, 2011. 

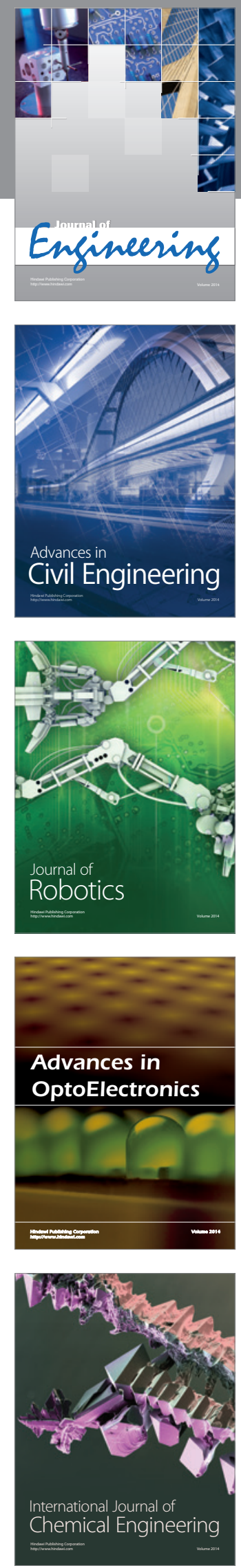

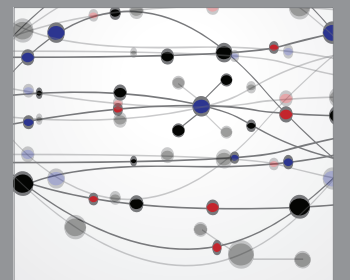

The Scientific World Journal
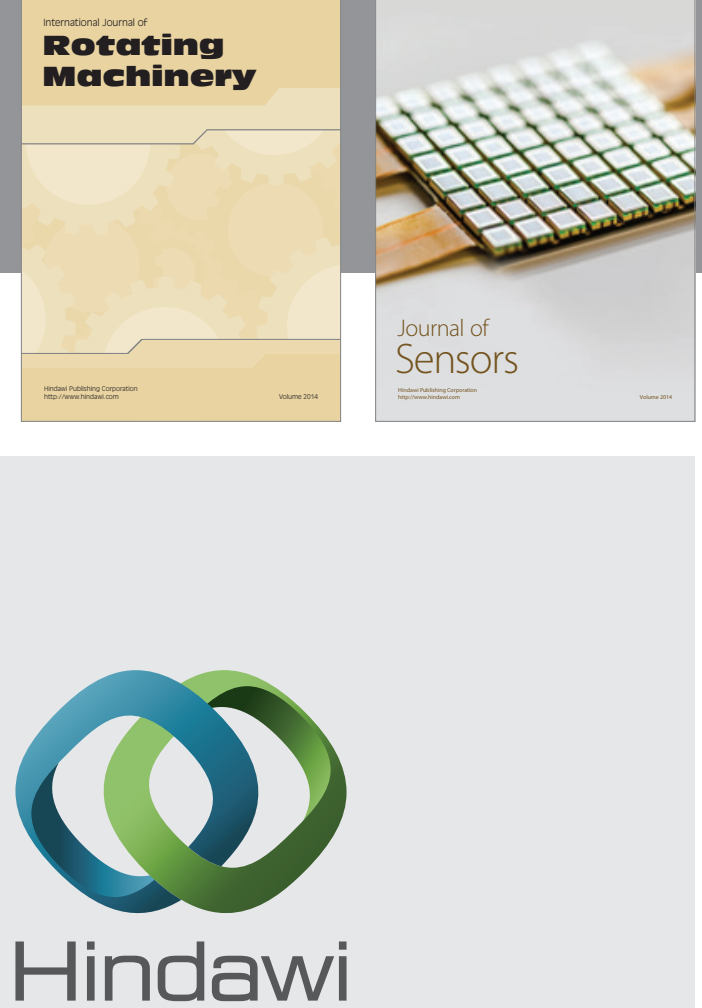

Submit your manuscripts at http://www.hindawi.com
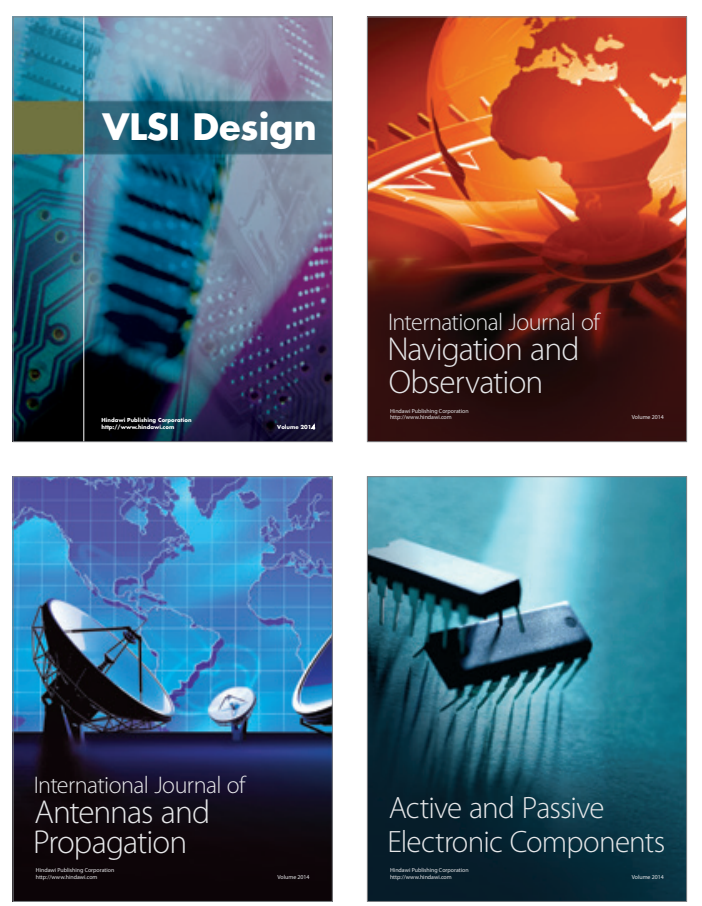
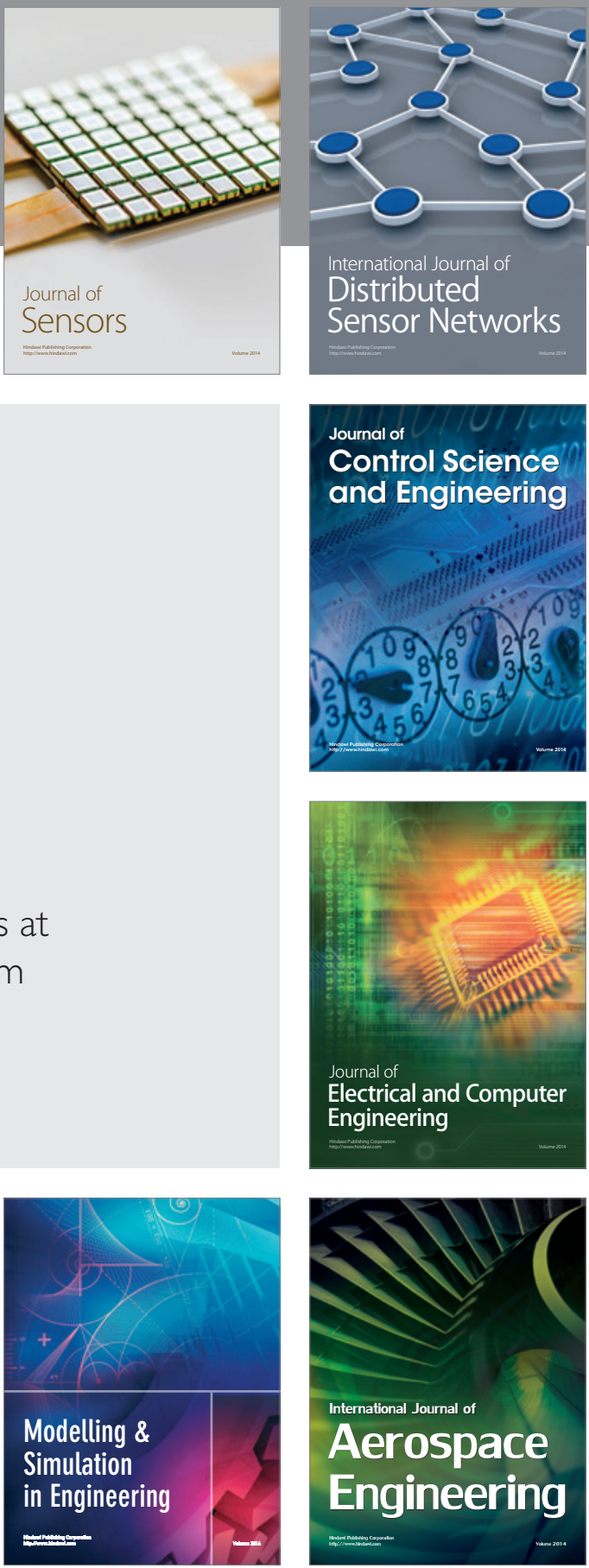

Journal of

Control Science

and Engineering
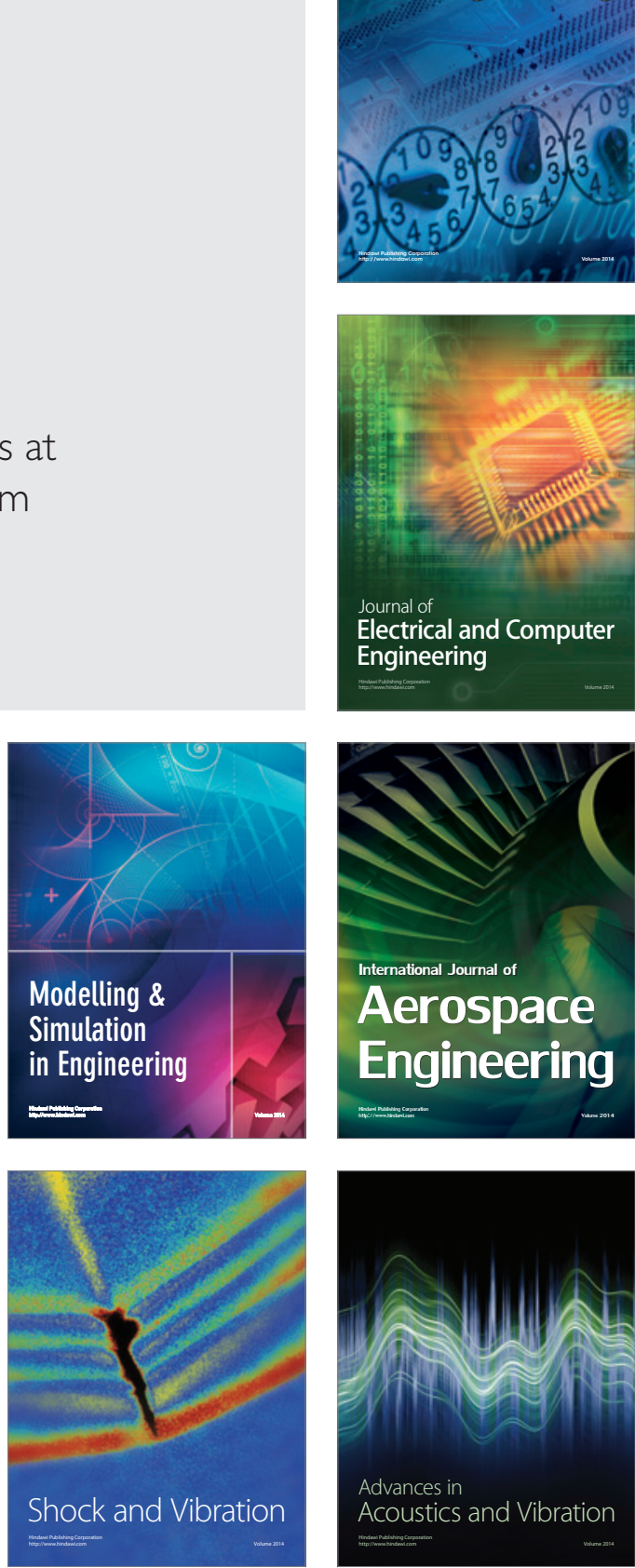